\title{
Interaction of Critical Care Practitioners With a Decision Support Tool for Weaning Mechanical Ventilation in Children
}

\author{
Silvia M Hartmann, Reid WD Farris, Ofer Yanay, Robert M DiBlasi, Christine N Kearney, \\ Joe D Zimmerman, Kristen Carlin, and Jerry J Zimmerman
}

\begin{abstract}
BACKGROUND: There is evidence that ventilator weaning protocols provide benefit to children receiving mechanical ventilation, but many protocols do not include explicit instructions for decreasing ventilator support from maximal settings. We evaluated care provider opinions on ventilator weaning recommendations made by a computerized decision support tool. METHODS: Recommendations for ventilator adjustment were generated using a computerized decision support tool based on the ARDSNet protocol using data from children with acute hypoxemic respiratory failure admitted to the pediatric ICU (PICU). Attending physicians, fellows, nurse practitioners, and respiratory therapists (RTs) caring for these patients answered a brief survey to assess whether recommendations were reasonable and whether the practitioner believed they could be implemented. RESULTS: RTs completed 99 surveys and ICU providers completed 96 surveys based on data from 10 patients. RTs and ICU providers found $63.9 \%$ and $65.3 \%$ of recommendations reasonable, respectively. There were 5 instances of disagreement between RTs and ICU providers. The percent of recommendations that RTs thought could be implemented was $29.9 \%$, whereas this figure for ICU providers was $26.3 \%$, with 4 instances of disagreement. Free-text responses indicated that many RTs and ICU providers were concerned about disrupting current patient stability and low tidal volumes. CONCLUSIONS: On initial evaluation, the decision support tool did not appear to be highly acceptable to RTs and ICU providers in our setting because recommendations were rarely implemented. In addition, acceptability did not increase over time as patients generally improved. Most respondents preferred to make no ventilator changes and felt the recommendations were too aggressive. The notable barrier to use was a perception of potential patient instability with weaning. Key words: mechanical ventilation; protocols; pediatric; acute hypoxemic respiratory failure; weaning. [Respir Care 2020;65(3):333-340. (C) 2020 Daedalus Enterprises]
\end{abstract}

\section{Introduction}

A comprehensive ventilator weaning protocol should include explicit rules to decrease support from maximum settings, predetermined screening criteria for a standardized extubation readiness trial, a standard method to perform an

\footnotetext{
Drs Hartmann, Farris, Yanay, and Zimmerman are affiliated with the Department of Pediatrics, Division of Critical Care Medicine, Seattle Children's Hospital, University of Washington School of Medicine, Seattle, Washington. Mr DiBlasi, Ms Kearney, and Mr Zimmerman are affiliated with the Department of Respiratory Care, Seattle Children's Hospital, Seattle, Washington. Ms Carlin is affiliated with the Children's Core for Biomedical Statistics, Seattle Children's Research Institute, Seattle, Washington.
}

The authors disclose a relationship with Seattle Children's Academic Enrichment Program. Mr DiBlasi discloses relationships with Vapotherm, Aerogen Pharma, Draeger Medical, Vero Biotech, and Neotech. extubation readiness trial, and a priori criteria for failure of the test. Ventilator weaning protocols in the adult and pediatric literature are often missing the first component of explicit rules to wean the ventilator until an extubation readiness trial can be performed. ${ }^{1-5}$ Prospective, interventional respiratory research could benefit from robust and validated ventilator weaning protocols, and patients could be liberated from mechanical ventilation sooner. Systematic reviews of both pediatric and adult populations suggest the clinical benefit of decreased duration of mechanical ventilation when ventilator weaning protocols are used. ${ }^{6,7}$ Prolonged mechan-

\footnotetext{
Correspondence: Silvia M Hartmann MD. E-mail: silvia.hartmann@ seattlechildrens.org.
}

DOI: $10.4187 /$ respcare.06877 


\section{Decision Support Tool for Mechanical Ventilation Weaning in Children}

ical ventilation beyond the period where it is medically necessary increases the risk for ventilator-associated complications, ICU stay, and cost. ${ }^{8-13}$ Researchers could use ventilator weaning protocols to minimize the variability in practice when comparing 2 interventions involving mechanical ventilation strategies.

A computerized decision support tool that was created for the pediatric ICU (PICU) population based on ARDSNet guidelines (www.ardsnet.org, Accessed August 21, 2019) ${ }^{14}$ has been evaluated in retrospective or hypothetical scenarios. Khemani and colleagues ${ }^{15}$ described that supplemental oxygen, peak inspiratory pressures, and mandatory ventilation rates were rarely weaned despite evidence of excessive oxygenation and hyperventilation in pediatric subjects with acute hypoxemic respiratory failure. In a recent survey completed by pediatric critical care fellows and attending physicians, the recommendations from this decision support software in 50 clinical scenarios were acceptable in $80 \%$ of instances. ${ }^{16}$ To our knowledge, there are no publications that evaluate the use of this decision support tool in real time.

Our primary hypothesis was that recommendations generated by the decision support software would be highly acceptable (ie, $>80 \%$ of recommendations would be considered reasonable) to ICU attending physicians, fellows, nurse practitioners, and respiratory therapists for patients in our ICU with acute hypoxemic respiratory failure receiving mechanical ventilation. Secondarily, we hypothesized that changes would be implemented by providers in $>80 \%$ of the instances that they found reasonable because the software suggests relatively modest changes for most scenarios. Finally, we hypothesized that suggested changes would be more likely to be implemented over time as lung disease generally stabilized and improved.

\section{Methods}

The institutional review board at Seattle Children's Hospital approved this prospective, descriptive, cohort study. We examined agreement between PICU providers and RTs and recommended ventilator weaning interventions generated by a software program using a brief survey instrument. The study was conducted in a free-standing academic children's hospital PICU where patients of $>44$ weeks corrected gestational age with both medical and surgical diagnoses are admitted. Critically ill neonates younger than 44 weeks corrected gestational age were excluded. Patients with primary diagnoses of congenital heart disease, dysrhythmia, or myocarditis cared for in the cardiac ICU were also excluded. All of the patients were supported using Dräger V500 series ventilators (Dräger, Lubeck, Germany). Attending physicians, fellows, nurse practitioners (collectively referred to as ICU providers) as well as RTs work in an environment that has adopted clinical standard work for specific diagnoses and equip-

\section{QUICK LOOK}

\section{Current knowledge}

Ventilator weaning protocols may provide benefit to children receiving mechanical ventilation, but many protocols do not include explicit instructions for decreasing ventilator support from maximum settings.

\section{What this paper contributes to our knowledge}

On initial evaluation, we did not find the decision support tool to be highly acceptable to respiratory therapists and ICU providers in our setting. There was no change in the acceptance of recommendations over a patient's course. Most respondents preferred no ventilator weaning or felt the tool provided recommendations that were too aggressive.

ment. Examples include a nurse-driven comfort protocol for analgesia and sedation for mechanically ventilated patients, a care pathway for patients with diabetic ketoacidosis admitted to the PICU, and a protocol for high-flow nasal cannula use in and outside of the PICU. At the time of this study, there existed institutional guidelines for extubation readiness trial screening, performance of the test, and failure of the extubation readiness trial, but there were no other protocols for ventilator management.

The study software was developed at Children's Hospital of Los Angeles for improved implementation of ARDSNet protocol principles for children with pediatric ARDS. The rules of the software have been modified by pediatric intensivists from the Collaborative Pediatric Critical Care Research Network (CPCCRN), a group of 8 children's hospitals that collaborate on multicenter investigations across a variety of topics (https://www.cpccrn.org, Accessed August 21, 2019). This software program includes escalation and weaning recommendations based on arterial oxygen saturations and $\mathrm{pH}$. Based on prior clinical experience, when oxygenation and ventilation goals have been achieved, the software recommends decreasing ventilator settings to maintain minimum $\mathrm{pH}$, tidal volume, and oxygen saturation goals.

Verbal consent was obtained from ICU providers and RTs to complete a brief survey after the generation of software recommendations. The fellows eligible for this study were restricted to those with $>6$ months of training. During the study period, 24 pediatric critical care attending physicians, 11 pediatric critical care fellows, and 4 pediatric critical care nurse practitioners comprised the care team eligible to take the survey. All 95 RTs in the hospital are trained to work in the PICU and were also eligible to take the survey. Written consent was obtained from subjects' families to use patient data to generate software 


\section{Decision Support Tool for Mechanical Ventilation Weaning in Children}

recommendations with the understanding that the PICU team would continue to manage the ventilator settings and were under no obligation to implement the software recommendations. Enrollment of mechanically ventilated subjects occurred between August 2015 and August 2016. Daily screening for patients was performed by 4 PICU attending physicians when they were not directly responsible for patient care. Written consent of the included subjects' families was obtained by the same 4 physicians. Inclusion criteria were anticipated need for invasive mechanical ventilation in the PICU for $\geq 48 \mathrm{~h}$ and suspected or documented acute lung process with severity that meets pediatric ARDS criteria. ${ }^{17}$ Initially, subject inclusion was restricted to those with moderate-to-severe pediatric ARDS who had acute onset of respiratory failure within 7 days, new infiltrates on chest radiograph, and oxygenation index $(\mathrm{OI}) \geq 8$ or oxygenation saturation index $(\mathrm{OSI}) \geq 7.5$ without suspicion for left heart failure or intracardiac shunt as the sole etiology for respiratory failure. ${ }^{17}$ This inclusion criterion was selected because these subjects are most likely to have repeat software recommendations generated over time and reflect the population for which the software recommendations should be the most easily applicable. With institutional review board approval, the OI/OSI criteria were relaxed throughout the course of the study to meet the goal of 15-20 subjects, which would provide 500 or more survey results. OI/OSI $\geq 6$ was used for broader inclusion of subjects. This threshold was selected because it has been used as a trigger for an extubation readiness trial in a multicenter randomized control trial of PICU subjects with lung disease. ${ }^{18}$ Exclusion criteria were congenital or acquired heart disease, congenital airway malformations, central hypoventilation syndrome, neuromuscular weakness, ongoing extracorporeal life support, limited resuscitation status, recent elective surgery (ie, within $5 \mathrm{~d}$ ), severe brain injury without spontaneous respiratory effort, intracranial hypertension, chronic mechanical ventilation, ward of the state or juvenile detention status, and adults $>21$ years of age. The exclusion criterion around postoperative status was also changed from 5 days after surgery to 2 days after surgery with institutional review board approval, although this criterion did not affect the enrollment of any subject.

Software recommendations were generated every $4 \mathrm{~h}$ after RTs performed standard, scheduled mechanical ventilator safety checks and charting in the electronic medical record. For this study, the software required separate input of ventilator settings, pulse oximetry saturations, capnometry values, and blood gas measurements. The bedside RT was responsible for entering these data. Printed instructions for data entry remained at the bedside with a separate study computer. Contact numbers for the principal investigator and RT coinvestigators were attached to the study computer to assist with data entry questions. Teaching sessions regarding the study and data entry were conducted at an RT staff meeting prior to study initiation. Just-in-time training on data entry by the consenting physician team was also available. After each data entry point, recommendations for adjustments to the ventilator were generated.

Recommendations regarding oxygenation and ventilation parameters were generated separately based on achieving adequate arterial oxygen saturations and $\mathrm{pH}$ from a blood gas, respectively. Our PICU providers generally obtain daily blood gases for management of ventilated patients during the weaning phase, which meant that there were many instances where no new blood gas information was available to generate recommendations. Alternatively, the software allows input of end-tidal values along with a baseline blood gas using several additional input steps to determine a relationship between the 2 values that can then be used calculate a $\mathrm{pH}$ and obtain ventilation recommendations. The bedside RT then completed a short survey with branching logic. Survey data were captured and evaluated through the secure web-based application RedCap (Research Electronic Data Capture), which is hosted by the University of Washington. ${ }^{19}$ The same software recommendations were then assessed by an ICU provider caring for the patient. RTs recorded their own survey answers and then called the ICU provider to discuss the software results and to input the provider's additional survey answers. This sequence was chosen to minimize interruptions in workflow.

The electronic medical record was reviewed for demographics, acute and chronic diagnoses, ventilator settings, vital signs, capnometry, blood gas results, duration of mechanical ventilation, outcome of extubation trials and subsequent need for respiratory support. Descriptive statistics, including frequencies and percentages, were calculated for whether the survey respondents felt the software recommendations were reasonable. Heat maps were used to assess patterns in agreement between the RT and the clinician with the computer software over the course of the subject's ventilator course. SAS 9.4 (SAS Institute, Cary, North Carolina) was used for all analyses.

\section{Results}

Verbal consent was obtained from all eligible PICU providers at staff meetings and fellow conferences. Verbal consent from all RTs was obtained at a department staff meeting. Written materials were provided by email to all participants.

Eleven patients admitted to the PICU were recruited. One subject was later found to have a diagnosis of neuromuscular weakness that met exclusion criteria and was excluded from the analysis. The median age of the remain- 


\section{Decision Support Tool for Mechanical Ventilation Weaning in Children}

ing 10 subjects was $12.5 \mathrm{y}$ (range 2 mo to $17 \mathrm{y}$ ). Six of 10 subjects were male. The most common acute diagnoses leading to admission were pneumonia (4 of 10 subjects) and septic shock ( 3 of 10 subjects). Three of 10 subjects had concurrent cardiac dysfunction or pulmonary hypertension that did not fully explain the degree of pulmonary impairment. Other acute diagnoses leading to ICU admission are noted in Table 1. All subjects had chronic medical conditions (Table 1), with the most common being chronic immunosuppression and static encephalopathy/developmental delay. Six of 10 subjects met criteria for severe pediatric ARDS and 2 of 10 subjects met criteria for moderate pediatric ARDS using OSI/OI criteria (https://www.cpccrn.org, Accessed August 21, 2019). The median OSI at recruitment was 7.7 (interquartile range [IQR] 6-11.9) for 9 of the 10 subjects. The time of enrollment was not recorded for a single subject, and we were unable to calculate the OI/ OSI at recruitment. The median worst OSI score within $24 \mathrm{~h}$ of intubation was 15.8 (IQR 9.7-20.2). This cohort had a median duration of mechanical ventilation of $5.5 \mathrm{~d}$ (IQR 4.8-10.6). This was similar to the median duration of mechanical ventilation for patients in the PICU during the previous year. One subject died before extubation was attempted, and upon review the death appeared to be unrelated to the study. The other 9 subjects underwent a trial of extubation with results shown in Table 1. One subject failed extubation immediately due to severe upper airway obstruction. Half of the remaining 8 subjects utilized high-flow nasal cannula, CPAP, or bi-level positive airway pressure support after extubation, which represents a combination of planned and rescue respiratory support.

Subjects were enrolled relatively early in their course of illness as a median of 79\% (IQR 60-87.7\%) of eligible ventilator checks occurred after enrollment. A median of $68.8 \%$ (IQR $60.1-91.4 \%$ ) of total ventilator checks were in mandatory ventilation modes compatible with software. The remaining $31.8 \%$ of ventilator checks were in the fully spontaneous mode (CPAP/pressure support ventilation). Survey data were obtained for $48.3 \%$ of possible evaluation points after enrollment. Complete survey data from both RTs and ICU providers were obtained for $46.8 \%$ of the possible assessments. Ninety-six out of 99 (97\%) surveys completed by RTs were also completed by an ICU provider. Pediatric critical care fellows completed $50.6 \%$ of surveys. First-year fellows with $>6$ months of training completed $13.7 \%$ of surveys. Second-year fellows completed $31.6 \%$ of surveys. Third-year fellows completed 5.3\% of surveys. Nurse practitioners completed $38.9 \%$ of surveys. Attending physicians completed $10.5 \%$ of surveys.

RTs reported that the software recommendations were reasonable in 62 of 97 surveys (63.9\%), which was similar to ICU providers who responded that the software recommendations were reasonable in 62 of 95 surveys (65.3\%).
There was a high level of agreement between RTs and ICU providers, as shown with the heat map in Figure 1. There were only 5 instances (5.3\%) where RTs and ICU providers had disparate opinions when both groups answered the survey. These disagreements are indicated by yellow boxes. There was no difference in the rate of agreement over the time of the study.

However, both groups felt that the recommendations should be implemented in the minority of instances. Respiratory therapists thought software recommendations could be implemented in 29 of 97 surveys (29.9\%). Physicians and nurse practitioners thought that software recommendations could be implemented in 25 of 95 surveys (26.3\%). There was a high degree of agreement between the 2 groups as illustrated with the heat map in Figure 2, with 4 instances (4.2\%) of disagreement shown in yellow. Again, there was no difference in the rate of agreement over the time of the study. When asked to describe the actions they would take as an alternative to the recommendations made by the software program, RTs and ICU providers most commonly responded that they would make no changes or that the software recommendations were too aggressive (Table 2).

All survey participants had the option of entering free text in response to why they would not implement the software recommendations. Themes that emerged from both the RT and PICU provider responses were a perception that patients were "unstable," concern that low tidal volumes in patients with adequate minute ventilation precluded further attempts at weaning, a desire to maintain current stability, and the limitation of the software program to include work of breathing as a factor in its recommendations. The perception of subject instability was the most frequent comment made by both groups, noted by RTs 7 times and by ICU providers 5 times. One example of such a comment is "Patient is not stable for weaning vent[ilator] settings at this time." No comments were explicit about the reason that a subject was judged to be unstable. Avoidance of further attempts at weaning the ventilator when tidal volumes were within the range of 4-6 mL/kg was cited 7 times by RTs and 3 times by ICU providers. An example of this idea was the comment that "recommended PIP (peak inspiratory pressure) wean when $\mathrm{V}_{\mathrm{T}}$ (tidal volume) was only $5 \mathrm{~mL} / \mathrm{kg}$." The desire to avoid changes and the potential increased work of breathing was noted 3 times by RTs and 5 times by ICU providers. Specific comments included: "Patient in good place; no need for change"; "Avoid too rapid weaning, make sure patient is tolerating the weaning done within the past $24 \mathrm{~h}$ "; "Too many weans in one day"; and "No further changes tonight." Current work of breathing limiting further ventilator weaning was reported 3 times by RTs and 2 times by ICU providers. 


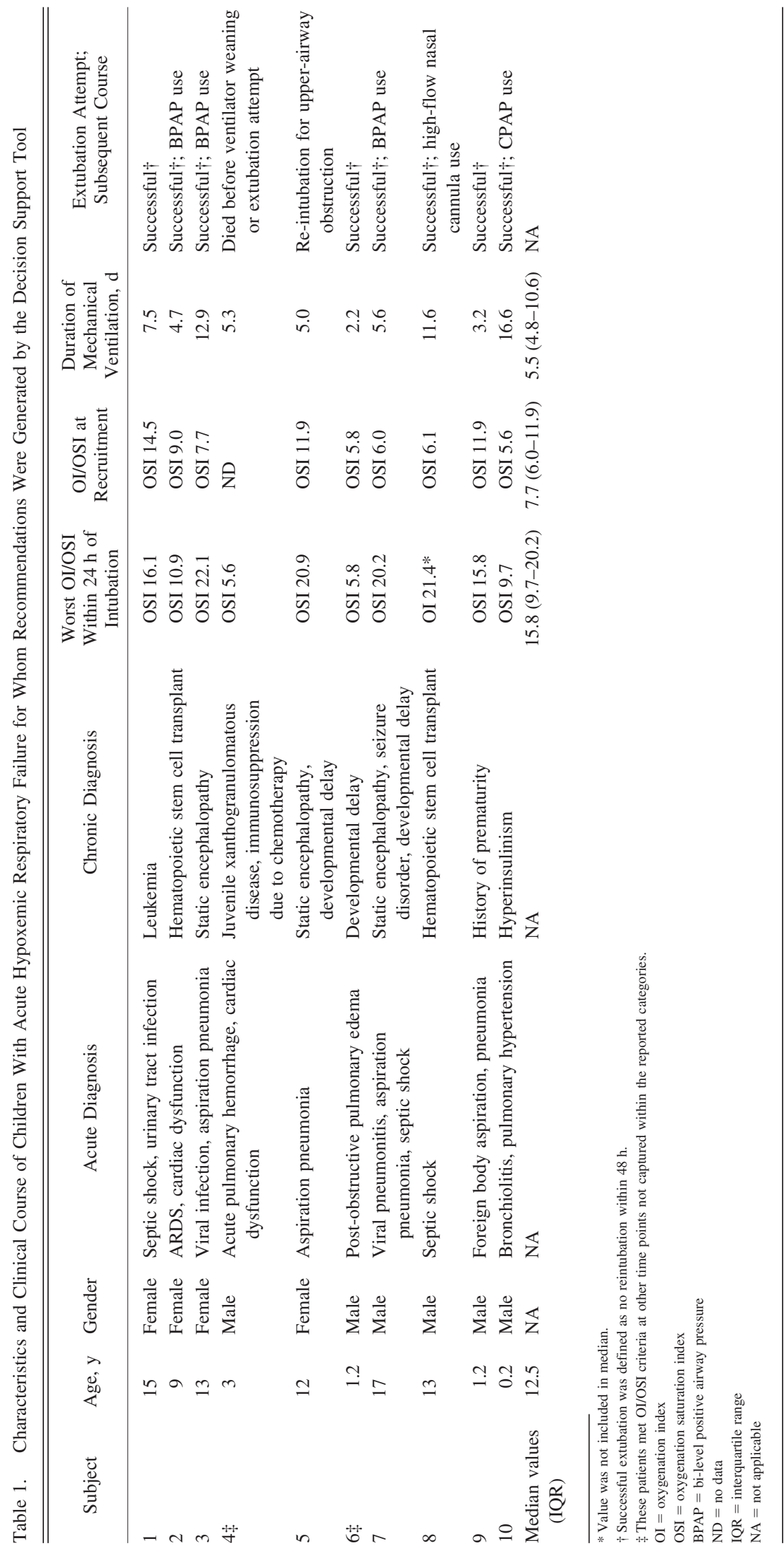




\section{Decision Support Tool for Mechanical Ventilation Weaning in Children}
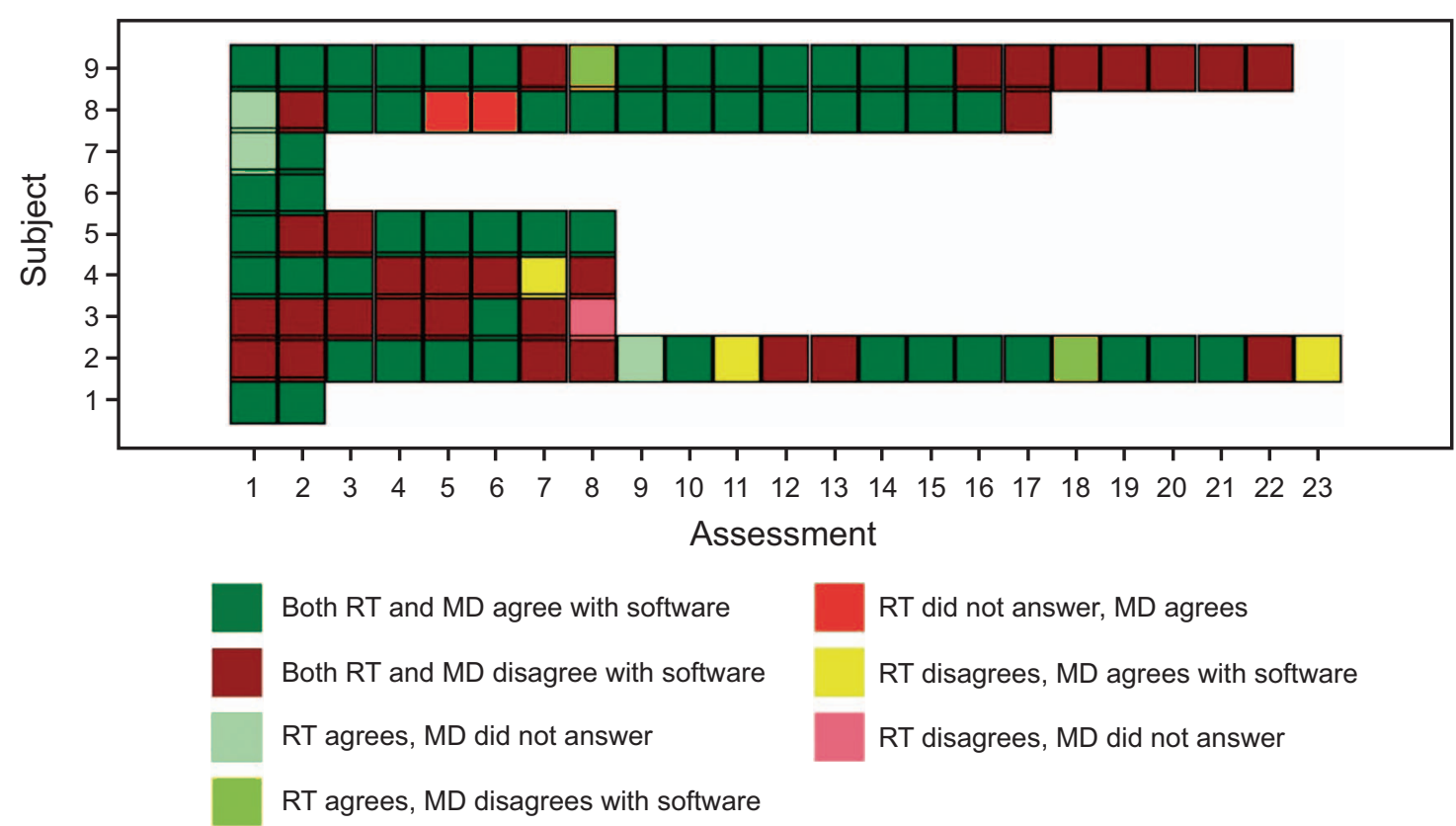

Both RT and MD agree with software

Both RT and MD disagree with software

RT agrees, MD did not answer

RT did not answer, MD agrees

RT disagrees, MD agrees with software

RT agrees, MD disagrees with software

Fig. 1. A heat map shows the responses of both respiratory therapists (RTs) and ICU providers (labeled as MD) to the question "Are the software recommendations reasonable?" for enrolled subjects over serial assessments, which indicate no change in agreement over time.
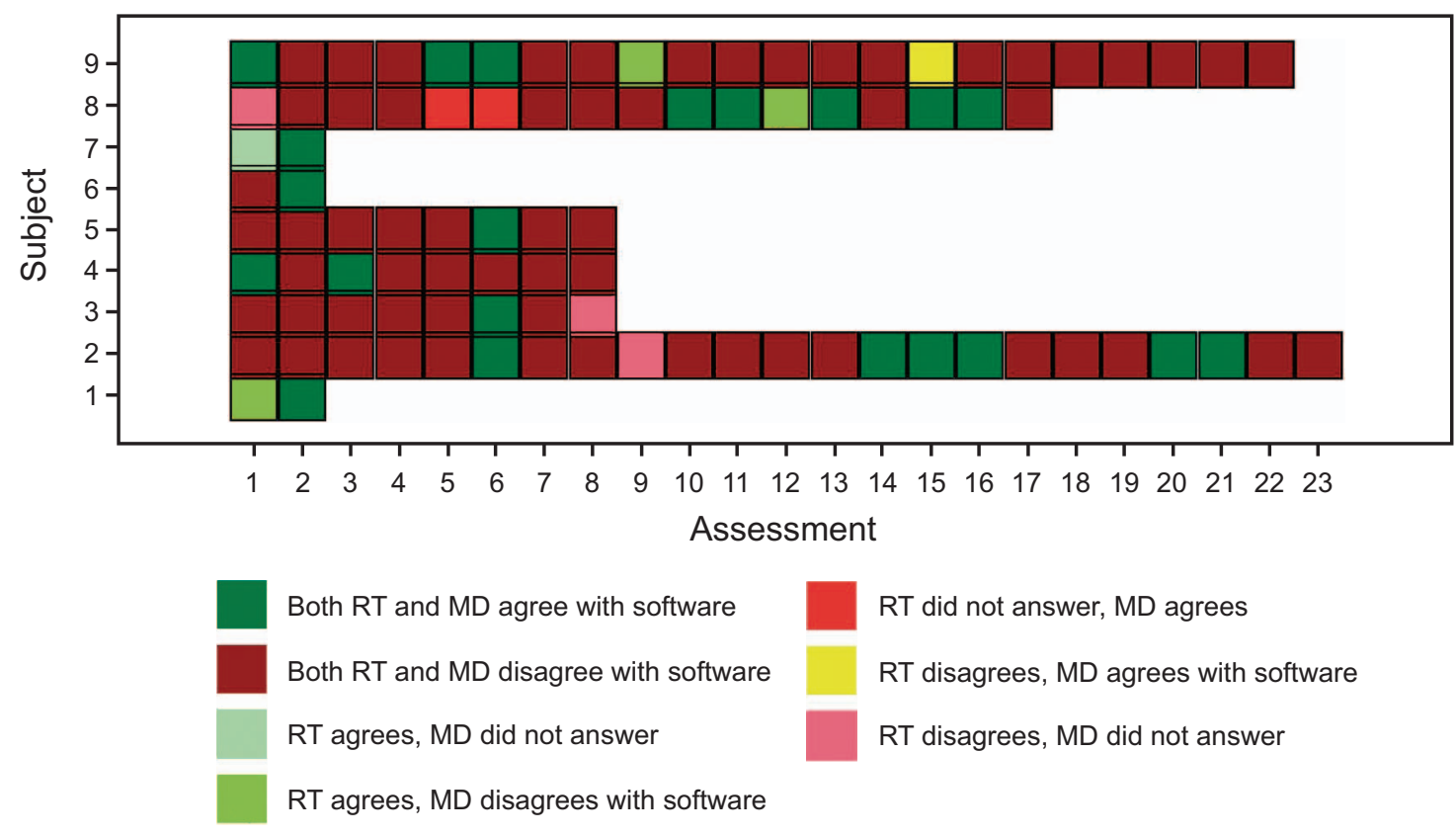

Both RT and MD agree with software

Both RT and MD disagree with software

RT agrees, MD did not answer

RT did not answer, MD agrees

RT disagrees, MD agrees with software

RT agrees, MD disagrees with software

RT disagrees, MD did not answer

Fig. 2. A heat map shows the responses of both respiratory therapists (RTs) and ICU providers (labeled as MD) to the question "Can you implement the recommendations now?" for enrolled subjects over serial assessments, which indicate no change in agreement over time.

\section{Discussion}

The major finding of our investigation is that RTs and ICU providers found the software recommendations reasonable in $64 \%$ and $65 \%$ of instances, respectively. This agreement rate fell short of our expectations. Furthermore, our secondary hypotheses were not supported. Decision support tool recommendations were not readily implemented by the team. Only $25-30 \%$ of recommendations were considered appropriate for implementation by the care team responding to the survey. The rate of implementation did not change over time, as shown with the heat 


\section{Decision Support Tool for Mechanical Ventilation Weaning in Children}

Table 2. Reasons for Declining to Implement Decision Support Tool Recommendations

\begin{tabular}{lcr}
\hline \hline & Respiratory Therapists & ICU Providers \\
\hline I plan on making different ventilator changes. & $8 / 66(12.1 \%)$ & $11 / 68(16.2 \%)$ \\
I believe the software recommendations are too aggressive. & $6 / 8(75 \%)$ & $6 / 11(54.5 \%)$ \\
I believe the software recommendations are not aggressive enough. & $0 / 8(0 \%)$ & $1 / 11(9.1 \%)$ \\
I prioritize weaning other variables. & $4 / 8(50 \%)$ & $6 / 11(54.5 \%)$ \\
I plan on making no ventilator changes. & $47 / 66(71.2 \%)$ & $39 / 68(57.4 \%)$ \\
Other & $15 / 66(22.7 \%)$ & $19 / 68(27.9 \%)$ \\
\hline
\end{tabular}

map analysis. We noted a significant difference between what was considered reasonable by members of the pediatric ICU team and what changes the team was willing to implement—at best, only $50 \%$ of reasonable recommendations would be implemented.

The greatest strengths of our study is that we simultaneously captured the opinions of RTs and ICU providers about the software recommendations at multiple time points in ventilator courses for subjects with pediatric ARDS, and we were able to generate a pragmatic view of how successful this software might be in our institution as part of a ventilator weaning protocol. By using real subject data that were evaluated by bedside providers, we noticed a significant decrease in the likelihood that providers and RTs would find recommendations reasonable and implement them compared to published survey data about hypothetical clinical scenarios. ${ }^{16}$ Another strength of our investigation was that most subjects met the definition of severe pediatric ARDS and represented a cohort of patients with relatively long ventilator courses, which provided many opportunities for evaluation with the software. Our cohort is one for which a software program based on ARDSNet guidelines is most applicable and recommendations are most likely to be congruent with previous education about management of patients with pediatric ARDS. We also had the opportunity to evaluate subjects during the majority of their ventilator course because almost $80 \%$ of possible evaluation points occurred after enrollment. Finally, we were able to record both RT and ICU provider perspectives for most of the captured evaluation points.

The most significant limitation of our study is the combination of slow enrollment with low numbers of subjects and the relatively low number of completed evaluations, which was just below 50\% of the total number of possible evaluations. A contributing factor that was hard to predict prior to initiating the study was the large number of patients who would have met enrollment criteria except that they were managed in an entirely spontaneous mode of ventilation, which was not a mode accommodated by the software. Although these patients would have otherwise met enrollment criteria, no software recommendations could have been generated, so they were not enrolled. Other limitations include the finding that the physicians answering the majority of surveys were trainees, generally in their first and second years of fellowship, and thus have less experience but the highest service obligation. The advanced practitioner program utilizing nurse practitioners at our institution also has a mix of experienced and inexperienced providers that mimics the spread of experience between fellows and attending physicians. Concurrent with the study were the introduction of a ventilator weaning lecture into the fellows didactic curriculum and the initiation of ICU Liberation, a quality-improvement initiative from the Society of Critical Care Medicine focused on weaning patients from the ventilator and performing extubation readiness trials as part of a bundle of care in the ICU. ${ }^{20}$ These other activities, which drew attention to ventilator weaning, likely did not affect the results of our study because there was no change in the frequency of ICU provider agreement or implementation of software recommendations over time. Likely the most important barrier to implementation of this clinical decision support tool was related to the challenges of change management within our environment.

We plan to examine the facilitators and barriers to ventilator weaning protocols more closely in our unit. This investigation highlighted some specific patient-related and protocol-related barriers. ${ }^{21}$ Patient-related barriers were most evident in the free-text comments about future patient decompensation with desaturation events or increased work of breathing that might result from the implementation of ventilator weaning. There was notable concern if tidal volumes were on the lower end of the acceptable range (ie, to $4 \mathrm{~mL} / \mathrm{kg}$ ) or if chest radiograph continued to be abnormal.

\section{Conclusions}

The most significant protocol-related barrier was that the software was cumbersome to use for an institution that does not obtain frequent blood gases. The additional steps needed to utilize end-tidal carbon dioxide measurements as a surrogate for carbon dioxide levels from a blood gas were numerous and challenging to perform correctly. We took several steps to improve the ease of use, such as a bedside guide with pictures and step-by-step instructions that were attached to the study laptop where data entry 


\section{Decision Support Tool for Mechanical Ventilation Weaning in Children}

occurred. Two RTs were available to answer questions during daytime and nighttime hours as "super users," although their familiarity waned over the course of the study due to the slow enrollment. Given that the software was created outside of our institution, it was impossible to adapt it for our particular needs.

The concept of ventilator weaning protocols is supported by the American Thoracic Society and American College of Chest Physicians in the most recent adult practice guidelines, ${ }^{22,23}$ although similar guidelines don't exist for pediatric patients. Guidelines can be translated into protocols in a multitude of ways to serve local environments. We examined the opinions of our ICU providers and RTs about a specific way to protocolize ventilator weaning and found a low rate of acceptance of the decision support tool recommendations. The software is based on ventilator management strategies for pediatric ARDS as vetted by pediatric intensivists, approximating best practices. Our results underscore the need to adapt protocols to address local barriers. ${ }^{24}$

\section{REFERENCES}

1. Jouvet P, Hernet P, Wysocki M. Development and implementation of explicit computerized protocols for mechanical ventilation in children. Ann Intensive Care 2011;1(1):51-60.

2. Ely EW, Baker AM, Dunagan DP, Burke HL, Smith AC, Kelly PT, et al. Effect on the duration of mechanical ventilation of identifying patients capable of breathing spontaneously. N Engl J Med 1996; 335(25):1864-1869.

3. Foronda FK, Troster EJ, Farias JA, Barbas CS, Ferraro AA, Faria LS, et al. The impact of daily evaluation and spontaneous breathing test on the duration of pediatric mechanical ventilation: a randomized controlled trial. Crit Care Med 2011;39(11):2526-2533.

4. Randolph AG, Wypij D, Venkataraman ST, Hanson JH, Gedeit RG, Meert KL, et al. Effect of mechanical ventilation weaning protocols on respiratory outcomes in infants and children. JAMA 2002;288(20): 2561-2568.

5. Esteban A, Frutos F, Tobin MJ, Alia I, Solsona JF, Valverdu I, et al. A comparison of four methods of weaning patients from mechanical ventilation. N Engl J Med 1995;332(6):345-350.

6. Blackwood B, Murray M, Chisakuta A, Cardwell CR, O'Halloran P. Protocolized versus non-protocolized weaning for reducing the duration of invasive mechanical ventilation in critically ill pediatric patients. Cochrane Database Syst Rev 2013;7:CD09082.

7. Blackwood B, Burns KEA, Cardwell CR, O'Halloran P. Protocolized versus non-protocolized weaning for reducing the duration of mechanical ventilation in critically ill adult patients. Cochrane Database Syst Rev 2014;11:CD006904.

8. Venkatachalam V, Hendley JO, Willson DF. The diagnostic dilemma of ventilator-associated pneumonia in critically ill children. Pediatr Crit Care Med 2011;12(3):286-296.

9. Srinivasan R, Asselin J, Gildengorin G, Weiner-Kronish J, Flori HR. A prospective study of ventilator-associated pneumonia in children. Pediatrics 2009;123(4):1108-1115.

10. Levine S, Nguyen T, Taylor N, Friscia ME, Budak MT, Rothenberg $\mathrm{P}$, et al. Rapid disuse atrophy of diaphragm fibers in mechanically ventilated humans. N Engl J Med 2008;358(13):1327-1335.
11. Jaber S, Jung B, Matecki S, Petrof BJ. Clinical review: ventilatorinduced diaphragmatic dysfunction-human studies confirm animal model findings. Crit Care 2011;15(2):206-214.

12. Bateman ST, Lacroix J, Boven K, Forbes P, Barton R, Thomas NK, et al. Anemia, blood loss, and blood transfusions in North American children in the intensive care unit. Am J Respir Crit Care Med 2008;178(1):26-33.

13. Chalom R, Raphaely R, Costarino AT. Hospital costs of pediatric intensive care. Crit Care Med 1999;27(10):2079-2085.

14. The Acute Respiratory Distress Syndrome Network. Ventilation with lower tidal volumes as compared with traditional tidal volumes for acute lung injury and the acute respiratory distress syndrome. N Engl J Med 2000;342(18):1301-1308.

15. Khemani RG, Sward K, Morris A, Dean JM, Newth CJ, NICHD Collaborative Pediatric Critical Care Research Network (CPCCRN). Variability in usual care mechanical ventilation for pediatric acute lung injury: the potential benefit of a lung protective computer protocol. Intensive Care Med 2011;37(11):1840-1848.

16. Sward KA, Newth CJL, Khemani RG, Page K, Meert KL, Carcillo JA, et al; on behalf of the Eunice Kennedy Shriver National Institute for Child Health and Human Development Collaborative Pediatric Critical Care Research Network (CPCCRN). Potential acceptability of a pediatric ventilator management computer protocol. Pediatr Crit Care Med 2017;18(11):1027-1034.

17. The Pediatric Acute Lung Injury Consensus Conference Group. Pediatric acute respiratory distress syndrome: consensus recommendations from the Pediatric Acute Lung Injury Consensus Conference. Pediatr Crit Care Med 2015;16(5):428-439.

18. Curley MA, Wypij D, Watson RS, Grant MJ, Asaro LA, Cheifetz IM, et al. Protocolized sedation vs usual care in pediatric patients mechanically ventilated for acute respiratory failure: a randomized clinical trial. JAMA 2015;313(4):379-389.

19. Harris PA, Taylor R, Thielke R, Payne J, Gonzalez N, Conde JG. Research electronic data capture (REDCap) - a metadata-driven methodology and workflow process for providing translational research informatics support. J Biomed Inform 2009;42(2):377-381.

20. Barnes-Daly MA, Phillips G, Ely EW. Improving hospital survival and reducing brain dysfunction at seven California community hospitals: implementing PAD guidelines via the ABCDEF bundle in 6,064 patients. Crit Care Med 2017;45(2):171-178.

21. Costa DK, White MR, Ginier E, Manojlovich M, Covidan S, Iwashyna TJ, Sales AE. Identifying barriers to delivering the awakening and breathing coordination, delirium and early exercise/mobility bundle to minimize adverse outcomes for mechanically ventilated patients: a systematic review. Chest 2017;152(2):304-311.

22. Girard TD, Alhazzani W, Kress JP, Ouellette DR, Schmidt GA, Truwit JD, et al; on behalf of the ATS/CHEST Ad Hoc Committee on Liberation From Mechanical Ventilation in Adults. An official American Thoracic Society/American College of Chest Physicians clinical practice guideline: liberation from mechanical ventilation in critically ill adults: rehabilitation protocols, ventilator liberation protocols, and cuff leak tests. Am J Respir Crit Care Med 2017;195(1):120-133.

23. Ouellette DR, Patel S, Girard TD, Morris PE, Schmidt GA, Truwit $\mathrm{JD}$, Alhazzani W, et al. Liberation from mechanical ventilation in critically ill adults: an official American College of Chest Physicians/American Thoracic Society clinical practice guideline: Inspiratory pressure augmentation during spontaneous breathing trials, protocols minimizing sedation, and noninvasive ventilation immediately after extubation. Chest 2017;151(1):166-180.

24. Jordan J, Rose L, Dainty KN, Noyes J, Blackwood B. Factors that impact on the use of mechanical ventilation weaning protocols in critically ill adults and children: a qualitative evidence synthesis. Cochrane Database Syst Rev 2016;10:CD011812. 